\title{
Offen für alle
}

\section{Stadtteilzentren für Demokratie und Vielfalt gegen rechtsextremistische und antisemitistische Einstellungen}

\author{
Eberhard Löhnert
}

Dr. Eberhard Löhnert ist Leiter der Geschäftsstelle Bezirke beim Paritätischen Wohlfahrtsverband, Landesverband Berlin e. V. E-Mail loehnert@paritaet-berlin.de
Die Berliner Stadtteilzentren sind in doppelter Weise für wirksame Ansätze gegen rechtsextremistische und rassistische Einstellungen prädestiniert: Mit ibrem kontinuierlichen Engagement für Demokratie und Toleranz erfüllen sie erstens einen wichtigen Arbeitsauftrag, zweitens erinnern sie damit an ibre eigenen Wurzeln.

Die ersten Nachbarschaftshäuser wurden schon Mitte der 1940er Jahre im Berlin der Nachkriegszeit gegründet. Neben einer Linderung der alltäglichen Not und dem Initiieren wichtiger Impulse für Selbsthilfeinitiativen, beispielsweise Suppenküchen und Nähstuben, spielten sie eine bedeutsame Rolle bei der Neuorientierung und Vermittlung von demokratischen Werten an die Bevölkerung. Dazu gehörten das Nachbarschaftsheim Schöneberg, das Nachbarschaftsheim Mittelhof in Steglitz-Zehlendorf, das Nachbarschaftsheim Neukölln und das Nachbarschaftshaus Urbanstraße in Friedrichshain-Kreuzberg.

Speziell diese auf Traditionen und Geschichte ausgerichteten Einrichtungen waren immer verlässliche Partner bei der Weiterentwicklung von Nachbarschaftsund Gemeinwesenarbeit in Berlin. Sie unterstützten den Aufbau weiterer Nachbarschaftseinrichtungen und Selbsthilfekontaktstellen in den 1980er Jahren in den Bezirken des ehemaligen West-Berlins sowie in den neunziger Jahren in den Bezirken des ehemaligen Ost-Berlins. Heute können wir sagen, Nachbarschaftsarbeit und Selbsthilfe sind gelebtes Engagement von Bürgerinnen und Bürgern nach den Prinzipien von Demokratie, Toleranz und gegenseitigem Respekt. Sie sind die Infrastruktur für bürgerschaftliches Engagement in unserer Stadt Berlin.

Dies wurde noch wichtiger, weil sich die Berliner Stadtteilzentren ab Mitte der 1990er Jahre zunehmend mit rechtsextremen und rassistischen Einstellungen auseinandersetzen mussten. Getreu ihrem
Motto »Offen für alle « setzen die Einrichtungen auf die Potenziale einer Vielfalt bei Religion, kultureller Herkunft, Weltanschauung und Lebensstilen ihrer Besucherinnen und Besucher. Damit schufen sie bewusst einen Gegenentwurf zu rechtsextremen Ideologien, in denen jeder seinen festgelegten Platz und seine festgelegte Rolle hat. Nachbarschaftsarbeit ist in diesem Sinne praktizierte Demokratie, denn sie bezieht alle Bürgerinnen und Bürger ein und fördert den gleichberechtigten Dialog sowie eine konstruktive Auseinandersetzung trotz oftmals konträrer Positionen und Meinungen. Damit bietet sie zugleich eine wirksame Alternative zu der Stigmatisierung von Menschen und gesellschaftlicher Ausgrenzung.

So ist es eine folgerichtige Entscheidung, dass Stadtteilzentren aktiv Impulse gegen Rechtsextremismus, Fremdenfeindlichkeit und Antisemitismus setzen und damit auf aktuelle Entwicklungen in den Bezirken reagieren.

Bei den letzten Wahlen zu den Berliner Bezirksparlamenten und dem Berliner Abgeordnetenhaus wählten 47.000 Wählerinnen und Wähler rechtextreme Parteien, doppelt so viele, wie in den Wahlen zuvor. In der Folge verstärkte sich die Präsenz rechtsextremer Gruppierungen in vielen Berliner Stadtteilen und die Zahl der Übergriffe auf Einrichtungen mit demokratischer und interkultureller Ausrichtung nahm zu. Als ein Brandanschlag auf das Haus Babylon im Stadtteil Hellersdorf verübt wurde, in Folge bereits der dritte Anschlag, nahm der Paritätische Wohlfahrtsverband in Berlin dies zum Anlass, gemeinsam mit den Berliner Stadtteilzentren das Jahr 2007 unter das Motto »Die Würde des Menschen ist unantastbar - NEIN zu Rechtextremismus, Rassismus und Antisemitismus « zu stellen. Prof. Barbara John, Vorsitzende des Paritätischen Berlin, übernahm die Schirmherrschaft. Die Initiative fand eine breite Unterstützung auf der politischen 
Ebene: Sämtliche Fraktionen im Berliner Abgeordnetenhaus, die zuständige Senatsverwaltung sowie Vertreterinnen und Vertreter der Bezirke wirkten im Beirat mit.

Die Initiative zielte vor allem darauf, Ideen und Engagement für demokratisches Handeln und demokratische Werte in den Stadtteilen Berlins zu fördern, Erfahrungen gleichberechtigter Teilhabe zu ermöglichen und die Achtung der Menschenwürde und Menschenrechte zu stärken, um latenten fremdenfeindlichen und antisemitischen Ressentiments wirksam zu begegnen.

Es beteiligten sich über 50 Berliner Stadtteilzentren mit mehr als 150 verschiedenen Projekten, darunter Ausstellungen, Diskussionsreihen, Filme, Theateraufführungen und eine Vielzahl von Aktivitäten mit Kindern und Jugendlichen. Die Initiative war vor allem deshalb ein großer Erfolg, weil sie entscheidend durch das aktive Engagement und die Kreativität von Bürgerinnen und Bürgern mitgetragen wurde. Es gelang in besonderer Weise, Netzwerke und Bündnisse in der Kooperation mit Schulen, Kitas und Einrichtungen der Jugendhilfe zu entwickeln. Die Stadtteilzentren gaben Impulse an weitere Einrichtungen in den Stadtteilen, sich ihres Beitrags für ein demokratisches Miteinander bewusster zu werden und nachhaltige Arbeitsansätze zu entwickeln, wie einige Beispiele zeigen:
- Babel e. V. setzt als einer der Hauptinitiatoren auf die Vielfalt und Verschiedenheit der Kulturen und Herkunft der Menschen, auf klare Zeichen gegen Rechtsextremismus und Rassismus, besonders bei jungen Menschen. So wirkte Babel e. V. zum Beispiel als Kooperationspartner der Initiative "Schule ohne Rassismus - Schule mit Courage « als Pate der Ernst-Haeckel-Schule in Hellersdorf. 70 Prozent der Schülerund Lehrerschaft hatten sich dort per Unterschrift $\mathrm{zu}$ den Prinzipien der »Schule ohne Rassismus « bekannt. Die jungen Menschen erlebten einen interkulturellen Dialog und das Zusammenleben im Haus Babylon. Aber: 30 Prozent der Schülerinnen und Schüler waren noch nicht bereit, klar Flagge für Demokratie und gegen Rechtsextremismus zu zeigen.

- Eine wirksame Initiative fand durch das Nachbarschaftshaus Pfefferwerk und die Selbsthilfekontaktstelle des $\mathrm{Hu}$ manistischen Verbands im Pankower Stadtteilzentrum statt: Die Geschichte des Hauses als ehemaliges jüdisches Kinderheim wurde aufgearbeitet, um sie in der Kooperation mit Schulen Kindern, Jugendlichen und Bewohnern im Bezirk näher zu bringen.

- Das Stadtteilzentrum »Villa offensiv« in unmittelbarer Nähe des Zentrums der Bundes-NPD und vieler ortsansässiger "freier Kameradschaften « enga- gierte sich bei der Umsetzung des regionalen »Festes der Demokratie «, an dem ein breites Netzwerk von Bürgerinnen und Bürgern, regionalen Einrichtungen und weiteren Akteuren mitwirkte. Danach übernahm der Verein die Registerstelle zur Dokumentation von rechtsextremen, rassistischen, antisemitistischen und homophoben Vorfällen und profilierte sich als Träger des Aktionsfonds zur finanziellen Unterstützung für Initiativen und Gruppen im Stadtteil, die sich für demokratische Werte aktiv einsetzen. »Offensiv 91 «zeigte: Außenwirksame Signale durch eine Organisation müssen immer auch intern konsequent umgesetzt werden. Das Nachbarschaftshaus wurde durch verschiedene Maßnahmen als "angstfreier Raum « und Anlaufstelle gegen die Präsenz rechtextremer Kräfte im Umfeld sichtbar gemacht. Durch einen Mitgliederbeschluss wurde der Bereich »Politische Bildung « in der Satzung verankert und es fanden Mitarbeiterschulungen $\mathrm{zu}$ den Themen Rechtsextremismus, Rassismus und Antisemitismus statt. An den Fortbildungen nahmen beispielsweise Erzieherinnen aus den Kitas des Trägers teil. »Offensiv 91 « ist heute selbst Träger des Zentrums für Demokratie im Bezirk Treptow-Köpenick. Dies zeigt die Wirksamkeit und Nachhaltigkeit der Initiative und ihrer Aktivitäten.

- Auf die Begegnung der deutschen und der türkischen Kultur setzte unter anderen das » Theater der Erfahrungen « des Nachbarschaftsheims Schönebergs mit seinem Projekt »Bunte Zellen«. Durch die interkulturell besetzte Theatergruppe wird nicht nur das Publikum angeregt, sich mit Vorurteilen, Gemeinsamkeiten, verschiedenen - und auch gleichen! - persönlichen Erfahrungen auseinanderzusetzen.

- Die Pankower Stadtteilzentren nahmen sich des Konflikts um den Moscheebau in Berlin-Pankow-Heinersdorf an. Hier galt es nicht nur, die Vorurteile in der Bevölkerung abzubauen, sondern auch darum, rechtsextremen und rassistischen Einstellungen den Nährboden zu entziehen sowie rechtsextremen Organisationen nicht das Handlungsfeld zu überlassen. Um den Dialog zwischen Menschen unterschiedlicher Positionen wieder auf einen Weg zu bringen und latent fremdenfeindlichen Einstellun- 
gen in der Bevölkerung entgegenzuwirken, führten die Pankower Stadtteilzentren eine Reihe von Gesprächen mit Heinersdorfer Bürgerinnen und Bürgern sowie Angehörigen der Ahmadyya-Gemeinde durch. Auch diese Initiative wirkte nachhaltig und hat sich $\mathrm{zu}$ einem »Bürgerverein für Demokratie und Nachbarschaft " weiterentwickelt, der heute ein Sprachrohr für die Bürgerinnen und Bürger in Heinersdorf ist.

\section{»Bunt statt braun«}

- Besonders wichtige Aktivitäten galten der Vernetzung und Bündelung von demokratischen Kräften im Stadtteil. Beispiele hierfür sind das Netzwerk »bunt statt braun « in Treptow-Köpenick oder das »Netzwerk gegen rechts « in Friedrichshain, an dem sich die Selbsthilfekontaktstelle Friedrichshain-Kreuzberg der Volkssolidarität aktiv beteiligt.

- Die vielfältigen Aktivitäten engagierter Bürgerinnen und Bürger in den Stadtteilzentren wurden von einem Filmteam ein Jahr lang begleitet und in einem Film mit dem Titel »jetzt erst recht « dokumentiert. Ganz bewusst räumt der Film der Darstellung rechtsradikaler Aktivitäten minimalen Platz ein. Im Vordergrund stehen beispielhafte Projekte, die die Menschen nachhaltig stärken in ihrem Selbstvertrauen und die ihre Fähigkeit fördern, sich mit Vorurteilen und Intoleranz kritisch auseinanderzusetzen und kulturelle Vielfalt als einen entscheidenden Wert in unserer Gesellschaft und als große Chance zu verstehen.

Die Initiative der Stadtteilzentren hat nicht nur berlinweit Aufmerksamkeit und Unterstützung erfahren. Der Film »jetzt erst recht " wurde vielerorts in den Berliner Stadtteilen gezeigt, aber auch im Berliner Abgeordnetenhaus oder der Bezirksverordnetenversammlung in MarzahnHellersdorf. Eine Delegation des Paritätischen und der Berliner Stadtteilzentren konnte den Film anlässlich eines Treffens von Nicht-Regierungsorganisationen am 6. Dezember 2007 in der UNO zum 60. Jahrestag der Verabschiedung der Allgemeinen Erklärung der Men- schenrechte übergeben. Eine Würdigung des Films wurde von den Organisatoren im Nachgang der Veranstaltung mitgeteilt. Auch während des Fachkongresses der Bundesarbeitsgemeinschaft der Freien Wohlfahrtspflege im Oktober 2008 wurde der Film gezeigt und diskutiert. Der Film kann kostenlos beim Paritätischen Berlin in der Geschäftsstelle Bezirke angefordert werden (E-Mail loehnert@paritaet-berlin.de).

\section{Resümee}

Die Einstellung »jetzt erst recht « prägte das Engagement aller an der Initiative Beteiligten und ist auch das Motto der kontinuierlichen Weiterentwicklung bei der Auseinandersetzung mit Rechtsextremismus und der Förderung demokratischer Werte. Der gezielte Umgang mit aktuellen Entwicklungen in Berlin wird jetzt noch bewusster in das Aufgabenfeld der Stadtteilzentren integriert. In diesem Sinne waren, sind und bleiben Stadtteilzentren in Berlin wichtige Orte für die Stärkung der Demokratie und unserer Zivilgesellschaft. Die Einrichtungen selbst entwickeln sich immer mehr zu Räumen, wo man sich Kraft und Unterstützung in diesem Prozess holen kann. Sie sind kompetente Impulsgeber für demokratische Mitgestaltung. Die Verstetigung der bewährten Aktivitäten und guten Erfahrungen bleibt eine Aufgabe für die Stadtteilzentren heute und morgen.

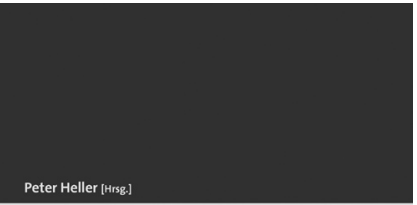

Innovative Funding Mechanisms for Social Change

$$
\text { Nomos }
$$

\section{Innovative Funding Mechanisms for Social Change}

Herausgegeben von Dr. Peter W. Heller

2009, 144 S., brosch., 24,- $€$, ISBN 978-3-8329-3948-9

Wo die soziale Sicherung des Staates versagt, finden Gemeinwohl-Unternehmer Lösungen, aber ihnen fehlen oft die notwendigen finanziellen Mittel.

Dieses Buch untersucht das Potential maßgeschneiderter sozialer Investmentfonds und Investitionsstrategien von Stiftungen, um auf neuen Wegen das fehlende Kapital bereitzustellen. Die Themen der portraitierten Finanzierungsmodelle reichen von der Augenheilkunde über die biologische Landwirtschaft bis zur Pressefreiheit. Die Autoren untersuchen die Voraussetzungen ihrer Entwicklung und ihre Relevanz für die Zukunft der Zivilgesellschaft.

\section{Nomos}

Bitte bestellen Sie im Buchhandel oder versandkostenfrei unter $\downarrow$ www.nomos-shop.de 